[Aus dem Kgl. Hygienischen Institut der Universität Erlangen.]

\title{
Gewerbehygienische Studien.
}

I. Über 0̈lschäden in Gewerbebetrieben.

Von

Prof. Dr. Wolfgang Weichardt und Dr. Hermann Apitzsch.

In der letzten Zeit traten in verschiedenen größeren Nürnberger Betrieben Erkrankungen auf, gegen welche Abhilfe im Interesse der Kriegsindustrie erwünscht war.

Die im folgenden beschriebene Erkrankung, eine Gewerbeakne infolge Beschäftigung mit nicht genügend gereinigten Ölen, machte sich besonders im vergangenen Jahre geltend, und die betroffenen Werke wandten sich um Abhilfe an das $K$. Hygienische Institut.

Besonders störend für die betreffenden Betriebe war es, daB bei dem bestehenden Mangel an Arbeitskräften manche der betroffenen Arbeiter und Arbeiterinnen infolge der aufgetretenen Erscheinungen es vorzogen, die Arbeit an den Maschinen zu verlassen und sich anderen Beschäftigungen zuzuwenden. Ungeschulte Arbeitskräfte mußten eingestellt werden, die vielfach die Arbeit wieder. verließen, nachdem auch bei ihnen die allenthalben siehtbaren Erscheinungen auftraten. Besonders empfindlich zeigte. sich der weibliche Teil der Arbeiterschaft.

Die Schwierigkeiten häuften sich, als unbegründete Gerüchte in Umlauf gesetzt wurden, daB eine übertragbare Krankheit die Ursacho des Leidens sei, daß die Säfte verderbt würden und ähnliches mehr.

Es war deshalb zunächst unsere Aufgabe, diese Gerïchte auf ihre Berechtigung zu prüfen und nach genauem Studium der krankheitserregenden Ursachen beruhigend einzugreifen, um zunächst den unbegründeten Arbeitsniederlegungen Einhalt zu tun. 
Zugleich bemühten wir uns, die Ursache des Leidens zu ergründen, um auf dieser Grundlage wirksame Vorbeugungsmittel ausfindig zu machen. Hierbei ließen wir uns im wesentlichen von praktischen Gesichtspunkten leiten. War doch in der Kriegszeit besonders der Umstand zu berücksichtigen, daß nur solche Aushilfsmittel in Frage kommen konnten, die leicht und billig $\mathrm{zu}$ beschaffen und vollkommen im Inlande herzustellen waren.

Die Schădigungen traten in unseren Betrieben vorwiegend bei Arbeitern der Metallindustrie auf. Es stellten sich bei solchen, die an automatischen Drehbänken und anderen ähnlichen Maschinen beschäftigt waren, nach etwa 12 Tagen, oder auch nach längerer Zeit, Hauterkrankungen, vor allen Dingen an den Vorderarmen, aber auch bei einigen im Gesicht, an (len Beinen und anderen Körperstellen ein.

Charakteristisch war das Auftreton zahlreicher, etwa stecknadelkopfgroßer, tiefschwarzer, an den Haarfollikeln lokalisierter Punkte, Comedonen. Völlig verschont blieben die Handflächen.

Zahlreiche Comedonen blieben längere Zeit bestehen, ohne eine weitere Entwicklung durchzumachen. Aus vielen entwickelten sich jedoch braunrötliche, erbsengroße, derbe perifollikuläre Knötchen, an deren Kuppe meistens eine kleine Pustel auftrat.

In diesem zweiten Entwicklungsstadium der Krankheit sah man dann zahlreiche papulopustulöse Effloreszenzen, die in ihrem Zentrum den Comedo erkennen ließen. Bei einzelnen Arbeitern waren diese Effloreszenzen auch an der Unterbauchgegend, am Gesäß, an den Beinen und besonders an den Unterschenkeln nachweisbar. Die Füße waren frei.

Eine spontanc Rïckbildung der Comedonen und Papeln ohne Narbenbildung schien nicht selten vorzukommen, jedoch waren auch kleine unauffällige Narben in dem Krankheitsbild in geringer Zahl nachzuweisen. Auch die Weiterentwicklung zu größeren Furunkeln wurde beobachtet.

Auffällig war, daß die Haare an den befallenen Gebieten brüchig wurden, ausfielen, und daß die Haarwurzeln teilweise gequollen waren.

Die Arbeiter klagten über mäßiges Jucken an den befallenen Körperstellen, immerhin konnten die direkten Belästigungen gering genannt werden. Allgemeine Krankheitserscheinungen haben wir nicht feststellen können, insbesondere war der Urin frei von Eiweiß.

Die auffälligen, auch für Laien sofort erkennbaren Veränderungen der Körperbedeckungen waren die Ursache der eingangs erwähnten Gerüchte und übertriebenen Anschauungen von der Schwere der Erkrankung.

Den Arbeitsniederlegungen, vor allem 'seitens der weiblichen Arbeiter, mußte im Interesse der Industrie unbedingt sofort entgegengearbeitet 
werden, und wir veranlaßten, nachdenı wir uns überzeugt hatten, dab eine an sich harmlose Gewerbeakne vorlag, die Betriebe, welche unsere Hilfe nachsuchten, zunächst durch Anschlag folgendes bekannt zu geben:

,In letzter Zeit sind infolge der Beschäftigung mit Ölen in der Kriegsindustrie Hauterscheinungen, vorwiegend an den Armen, aufgetreten.

Diese Hauterseheinungen sind harmloser Natur.

Eine allgemeine Gesundheitsschädigung, insbesondere eine Erkrankung des Blutes oder der inneren Organe ist nicht zu befürehten.

Auch handelt es sich nicht um eine übertragbare Krankheit, die von Person zu Person ansteckend wirkt.

Um die Hauterscheinungen zu vermeiden, empfiehlt sich die Reinhaltung des Körpers, vor allem der der Einwirkung der Öle direkt ausgesetzten Teile der Arme.

Die Arme sollen nach beendeter Arbeit, bei Beginn der Mittagspause und beim Verlassen der Fabrik, mit warmem Wasser und Seife gründlich gereinigt werden.

Weitere Maßnahmen zur Verhiitung der Hauterscheinungen sind in die Wege geleitet."

Nach den eingezogenen Erkundigungen waren die Erkrankungen in unseren Betrieben vor dem Kriege nur ganz gelegentlich beobachtet worden. Als Erklärung für die auffällige Häufung in den letzten Jahren wurde von vielen Leitern der betreffenden Fabriken und auch den Öl liefernden Firmen die nicht genügende Reinigung der verwendeten Mineralöle herangezogen. Diese nicht genügende Reinigung wurde auf den derzeitigen Mangel an Schwefelsäure zurückgeführt. . Das Öl wurde in unseren Betrieben beim Verarbeiten der Drehstücke usw. verwendet. Es spritzte zum Teil bei der Arbeit von den Masehinen, so daß nicht nur die Vorderarme, sondern auch das Gesicht vielfach mit Öl beschmutzt wurden. Ferner fanden wir auch allenthalben die Kleider, vor allem die Beinkleider von Öl durchtränkt.

In einem Betriebe hatte man schon vor unsercm Eingreifen Schutzärmel zu verwenden versucht, jedoch ohne Erfolg, was wegen der ungeeigneten Beschaffenheit der das öl aufnehmenden Ärmel erklärlich war. Ferner hinderten die Ärmel, vor allem wenn sie ölhaltig geworden waren, die Leute bei der Arbeit außerordentlich.

Unser Krankheitsbild war am meisten dem ähnlich, welches als Petroleumakne in der Literatur bekannt ist. Chlor, das auch zu Akneformen Veranlassung gibt, und worüber wir vor allem K. B. Lehmann ${ }^{1}$

1 K. B. Lehmann, Studien über Chlorakne. Archiv /. Hygiene. 1903. S. 322 . 
und Anderen ${ }^{2}$ Untersuchungen verdanken, kam bei unseren Arbeitern nicht in Betracht.

Uber allgemeine und Hautvergiftungen durch Petroleum hat L. Lewin $^{2}$ bereits im Jahre 1888 Untersuchungen ausgeführt und auch über ihre Entstehung einige Uberlegungen angestellt. Lewin fand, dab besonders die schweren Petroleumbestandteile die Eigenschaft besitzen, tierische Gewebe in Entziundungen zu versetzen. Besonders waren es die bei 250 bis $360^{\circ}$ destillierbaren Petroleumprodukte, welche wirksam waren. Lewin führte diese Produkte bei seinen Versuchen in den Magen ein und setzte die dort sich zeigenden Veränderungen in eine Parallele mit den an der Haut der Arbeiter sich abspielenden Prozessen. Besonders die Verstopfung der Follikelmündungen, der Haut- und Talgdrïsen durch das schmierige Petroleumprodukt müssen nach Lewin zu Veränderungen führen. Ferner die Mehrbildung von Sekreten und die gleichzeitige Behinderung der Exkretion, welche eine Erweiterung des Ausführungsgangs nach sich ziehen.

Wir haben die Entzündung erregenden Eigenschaften unserer öle nicht mittels intrastomachaler Einverleibungen verfolgt, sondern wir bedienten uns der Ohren albinotischer Kaninehen, indem wir gereinigtes und ungereinigtes Öl auf sie brachten und dann den Grad der Entzündung, vergleichsweise maßen.

Aus dem Pustelinhalt der befallenen Haut der Arbeiter gelang es uns, neben anderen Eitererregern, auffallend viel Streptokokken zu züchten. Es ist wohl die Annahme berechtigt, dab beim Zustandekommen des charakteristischen Krankheitsbildes beim Menschen verschiedene Ursachen wirksam sind. Vielleicht wird durch die anfänglichen Reizungen lokaler Partien infolge dauernder Wirkung der nicht indifferenten Mineralöle die Widerstandsfähigkeit gegen infektiöse Prozesse allmählich herabgesetzt, so daß sekundär Eitererreger und ihre Spaltprodukte beim Zustandekommen der lokalen Herde mit beteiligt sind.

Das uns übersandte Öl war von dickflüssiger Beschaffenheit, dunkler Farbe, schwachblauer Fluoreszenz und von unangenehmem Mineralölgeruch.

Wie die Prüfung ergab, waren freie Säuren oder Alkalien in irgend erheblichen Mengen nicht darin vorhanden. Einige Kubikzentimeter des Rohöls wurden in einem Gemisch gleicher Teile Äther und Alkohol gelöst

1 Die recht ausgedehnte Literatur über diesen Gegenstand ist in den bekannten Sammelwerken, auf welche hier verwiesen sei, übersichtlich zusammengestellt.

2 L. Lewin, Ưber allgemeine und Hautvergiftung durch Petroleum. Virchows Archiv. CXII. 1888. 
und mit Phenolphthalein versetzt. Auf Zusatz eines Tröpfchens Normalalkali trat Rötung ein.

Das unbehandelte Öl wurde auf das Ohr eines Kaninchens (Albino) aufgetragen. Schon im Laufe von 24 Stunden trat erhebliche Rötung des Ohres ein. Diese steigerte sich nach wiederholtem Auftragen des Öles während der nächsten Tage, und es kam sogar zu Exsudationen. Dic Haare über den eingeriebenen Stellen schwanden, und dieser Haarschwund zeigte sich auch auf dem Rücken, entsprechend der. Lage des mit Öl bestrichenen Ohres. Auch an der daselbst befindlichen Haut zeigten sich schließlich hochgradige Rötung und Exsudationen.

Gleichzeitig wurden Kontrollversuche angestellt, um den mechanischen Effekt der Öleinreibung selbst kennen zu lernen. Es wurden die Ohren von gleich jungen Albinos mehrmals am Tage mit reinem OlivenöP eingerieben. Wie zu erwarten, traten keinerlei Reizerseheinungen auf.

Ehe wir an eine Entfernung der in dem öl offenbar vorhandenen reizenden Substanzen gingen, versuchten wir, ob es möglich sei, ähnliche Reizerscheinungen durch wiederholtes Bestreichen der Ohren mit Paraffinum liquidum, also einem reinen Mineralöle, hervorzubringen. War es doch immerhin möglich, daß die länger dauernde Bedeckung der Hautstelle mit Mineralölen allein schon einen sclädigenden Einfluß ausübte. Unsere dahin angestellten Versuche belchrten uns, daB reines Paraffinum liquidum, mehrmals auf das so empfindliche Ohr der Kaninchen gestrichen, keine Reizerscheinungen hervorrief.

Es mußte sich also bei den zu untersuchenden ölen um besondere, infolge ungenügender Reinigung zurückgebliebene, reizende Substanzen handeln, auf welche die zirkumskripten Entzündungserscheinungen zurückzuführen waren.

Das stärkere Autreten der Hauterscheinungen war mit den durch den Krieg bedingten wirtschaftlichen Verhältnissen in Zusammenhang zu bringen. Nach den eingezogenen Auskünften waren Hautschädigungen der beschriebenen Art in unseren Betrieben vor dem Kriege nur ganz vereinzelt beobachtet worden, während jetzt ihr Auftreten sich häufte. Die Öle stammten von verschiedenen Bezugsquellen. Zugleich wurde darauf hingewiesen, daß infolge des Fehlent von Schwefelsäure die Reinigung der jetzt verwendeten Öle nicht mehr so sorgfältig wie früher durchgeführt würde. Da, wie wir gesehen haben, das Paraffinum liquidum Reizerscheinungen nicht hervorrief, "glaubten wir zunächst die zweifellos in unseren Ölen vorhandenen harzigen Bestandteile dafür verantwortlich machen zu können. War es doch möglich, daß gerade derartige Harze infolge ihrer physikalischen Beschaffenheit zu einer Verstopfung der Haut- 
poren fühtren konnten, die zweifellos von schädlichem Einfluß sein mußte. Bestärkt wurden wir in dieser Annahme durch die Eigenschaft des verwendeten Öles, Fette leicht zu lösen. Es gelang uns z. B., Lanolin und Schweinefett in beliebigen Mengen in dem öle aufzulösen. Der Vorgang konnte also immerhin so sein, daß primär eine Auflösung des Hauttalges eintrat, und daß sich dann sekundär harzige Bestandteile in den Talgdriisen festsetzten.

Um einen Einblick in diese Verhältnisse zu bekommen, bestimmter wir zumächst den Harzgehalt der öle.

Der Harzgehalt von Mincräölen läßt sich bekinntlich durch Ausschütteln mit konzentrierter Schwefelsäure in Petrolätherlösung in eincm graduierten Gefäß leicht ermitteln.

Nach Kooper schüttelt man das zu untersuchende ÖI mit konzentrierter Schwefelsäure in Petrolätherlösung und kann an der Volumzunahme der konzentrierten Schwefelsäure direkt den Harzgehalt ablesen. Kooper nimmt diese Bestimmung in einem eigens von ihm angegebenen Röhrchen vor, in dem dạs öl, die Schwefelsäure und der Petroläther zuerst gut durchgeschüttelt und dann zentrifugiert werden.

Nach Kooper sehwankt der Harzgehalt der Mineralöle im allgemeinen zwischen 5 und 25 Prozent und beträgt bei guten Ölen meist 3 bis 12 Prozent des Volumens des Öles. Da uns die Kooperschen Röhrehen nicht zur Verfügung standen, nahmen wir die Messung in graduierten Zylindern vor und konnten nach mehrtägigem Stehenlassen konstante Ablesungen vornehmen.

So wurden $5 \mathrm{ccm}$ konzentrierter Schwefelsäure, $10 \mathrm{ccm}$ Ol, etwa $22 \mathrm{ccm}$ Petroläther in einem $100 \mathrm{ccm}-Z y l i n d e r$ gut durchgeschüttelt. Nach mehrstündigem Stehen hatte die Schwefelsäure ungefähr um einen Kubikzentimeter zugenommen. Der Versuch wurde wiederholt mit genau $3 \mathrm{ccm}$ Schwefelsäure, $6 \cdot 2 \mathrm{ccm}$ öl und $15 \cdot 8 \mathrm{~cm}$ Petroläther in einem geeichten engen $25^{\circ} \mathrm{ccm}-Z y l i n d e r$. Nach mehrtägigem Stehen hatte sich die schwarze Schwefelsäure fast quantitativ abgeschieden, sie betrug $3.5 \mathrm{ccm}$, hatte also um $0.5 \mathrm{ccm}$ zugenommen. Die angewandten $6.2 \mathrm{ccm}$ Öl hatten demnach $0.5 \mathrm{ccm}$ Harz enthalten oder rund 8 Prozent. Nach dieser beiläufigen Messung überstieg also der Harzgehalt nicht den eines guten Mineralöles, so daß die Schädlichkeit wohl kaum auf zu große Mengen von Harzen in den verwendeten ölen zurückzuführen war.

Daß die reizenden Eigenschaften der Öle überhaupt wohl nicht auf die Harze als solche zurïckzuführen sind, zeigten uns die Versuche, bei denen wir die in den Mineralölen zurïckgebliebenen Harze sollkommen entfernten: 
Wir versuchten zunächst, um die bei den jetzigen Verhältnissen zu teuere Schwefelsäurebehandlung zu vermeiden, durch Schütteln mit Tierkohle, Talkum und Kieselgur, die ja bekanntlich infolge ihrer absorbierenden Eigenschaften in größtem Maßstabe als verhältnismäßig billige Reinigungsmittel technische Verwendung finden, Harze und bzw. andere schädliche Substanzen zu entfernen.

Die vollständige Entfernung der Harze mit praktisch anwendbaren Mengen dieser Mittel gelang nicht, obgleich die öle bedeutend heller, der Geruch schwächer und weniger unangenehm geworden war.

Auch zeigten die angestellten Tierversuche; da $B$ dic reizenden Substanzen keineswegs aus den Ölen verschwunden waren. Die bestrichenen Ohren unserer Albinos waren sehr bald stark gerötet, und Exsudationen traten ein.

Wir entfernten die Harze nun vollständig durch Behandeln mit konzentrierter Schwefelsäure:

Das Verfahren war folgendes:

1. $30 \mathrm{ccm}$ Öl wưden mit $20 \mathrm{ccm}$ konzentrierter Schwefelsäure du'chgeschüttelt, dabei trat starke Verharzung ein, während das Reaktionsgemisch deutlichen Geruch nach schwefliger Säure zeigte. Nach öfterem käftigen Schütteln, darauffolgendem guten Auswaschen, zuerst mit Wasser, dann mit verdünnter Natronlauge, wieder folgendem Auswaschen mit Wasser und schließlichem Trocknen mit Chlorkalzium und Filtrieren wurde ein helles Öl von gelber Farbe mit schwachblauer Fluoreszenz und nur ganz schwachem an Petroleum erinnernden Geruch erhalten.

2. $50 \mathrm{ccm}$ öl wurden mit nur $5 \mathrm{ccm}$ konzentrierter Schwefelsäure, sonst in gleicher Weise, behandelt. Die Farbe des zweiten Endprodulstes war etwas dunkler als die des ersten. Die Ausbeute an gereinigtem öl betrug bei der zweiten Portion ungefähr 60 Prozent des angewandten Rohöls.

Bei den angestellten Tierversuchen zeigte es sich nun, daß auch durch diese Behandlung die reizenden Substanzen aus dem Öle noch nicht verschwunden waren, selbst die mit verhältnismäßig viel Schwefelsäure behandelte Probe reizte noch erheblich, wenn auch eine Besserung dem Rohöl gegénüber nicht zu verkennen war.

Wir mußten deshalb unsere ursprüngliche Annahme und die oben entwickeiten physikalischen und chemischen Anschauungen uiber den Vorgang des Reizungsprozesses fallen lassen. Die Harze als solche waren zweifellos nicht für den Reizeffekt verantwortlich zu machen.

Der bei der Behandlung mit konzentrierter. Schwefelsäure auftretende starke Geruch nach schwefliger Säure wies daraif hin, daß in dem Öle 
unverhältnismäßig viel leicht oxydierbare Substanzen, d.h. ungesättigte Verbindungen vorhanden sein mußten. Von großem Interesse für unsere Untersuchung waren die bekannten Arbeiten von Paal. ${ }^{1}$ Dieser zeigte, daB durch reduzierende Behandlung selbst des so giftigen Krotonöls dessen reizende Wirkungen beseitigt werden konnten.

Paal bediente sich für diese Versuche katalytischer Reduktion und benutzte kolloidale Metalle der Platingruppe als Wasserstoffüberträger. Aus den Paalschen Untersuchungen geht hervor, daß durch Beseitigung der ungesättigten Verbindungen, bzw. durch Umwandlung ungesättigter in gesättigte Verbindungen, auch die Giftwirkung aufgehoben wird. Derselbe Erfolg mußte sich durch Oxydation der in den Mineralölen vorhandenen ungesättigten Verbindungen erreichen lassen oder durch deren Umwandlung in gesättigte Verbindungen, wie sie durch Halogenaddition an ungesättigte meist leicht zu erreichen ist.

Wir wandten zunächst Kaliumpermanganat und zwar in Verbindung mit Salzsäure an.

$30 \mathrm{cem}$ Öl und $60 \mathrm{cem}$ konzentrierter Salzsäure wurden in einem Kolben gut durchgeschüttelt, dabei trat bereits Erwärmung ein, harzige Flocken setzten sich an der Wand des Kolbens fest. Von diesen wurden das ÖlSäuregemisch nach mehreren Stunden in einem Scheidetrichter abgegossen. Dann wurde nach und nach von einer wässerigen Permanganatlösung ( $5 \mathrm{~g}$ in $100 \mathrm{ccm}$ Lösung) zugegeben, und das Ganze gut durchgeschüttelt, bis der unangenehme Ölgeruch verschwunden, dagegen deutlicher Chlorgeruch vorhanden war. Verbraucht wurden $50 \mathrm{~cm}$ Permanganatlösung. Nach einigen Stunden Stehen hatten sich das Öl und die braune wässerige Lösung so weit getrennt, daß die letztere bequem vom öl abgegossen werden konnte. Hierauf wurde das Öl im Scheidetrichter 3 mal mit Wasser durchgeschüttelt, dann 3 mal mit stark verdünnter Natronlauge und schließlich nochmals 5 mal mit Wasser gewaschen.

Dann wurde das Öl mit Chlorkalzium getrocknet, filtriert und nochmals gründlich mit Kaliumkarbonat getrocknet und filtriert. ZurüGkerhalten wurden $22 \mathrm{ccm}$ Öl. Es hatte nur ganz schwachen nicht unangenehmen Geruch, rotbraune Farbe und war dickflüssig. Das öl zeigte keine Fluoreszenz mehr. Diese Versuche ermutigten uns, diesen Weg weiter zu beschreiten, und wir versuchten, ähnliche Wirkungen durch Behandlung mit Chlorkalk zu erzielen und von der verhältnismäßig teuren Permanganatbehandlung sund Salzsäure abzusehen.

In der Tat gelang es, ein den Anforderungen der Praxis genügend entsprechendes Reinigungsverfahren auf diesem Wege ausfindig zu machen:

\footnotetext{
1 Paal, Ber. d. deutschen chem. Ges. Bd. XLII. 1909. S. $1541 \mathrm{ff}$.
} 
$50 \mathrm{ccm}$ Öl wurden in einem Schüttelzylinder mit $15 \mathrm{~g}$ Chlorkalk gut durchgeschüttelt. Wir ließen das Gemisch 15 Stunden stehen und schüttelten es während dieser Zeit häufig durch. Dann wurde vom Chlorkalk in einen Schüttelzylinder abfiltriert, wobei $40 \mathrm{ccm}$ öl zurückerhalten wurden. Diese $40 \mathrm{ccm}$ wurden in dem gleichen Schïttelzylinder mit $40 \mathrm{ccm}$ Wasser durchgeschüttelt und dies mehrmals wiederholt. Das Wasser wurde abgegossen, und das zurückbleibende Öl mit Kaliumkarbonat getrocknet und filtriert. Zurückerhalten wurden etwa $40 \mathrm{ccm}$. Das Öl war von rotbrauner Farbe und etwas heller wie das mit Permanganat behandelte. Es roch nicht unangenehm und zeigte keine Fluoreszenz.

Während das öl vor der Behandlung mit Permanganat und Salzsäure bzw. mit Chlorkalk bei der Prüfung auf Halogene mittels der Beilsteinschen Probe (Erhitzen mit Kupferoxyd) kein positives Resultat gab, gelang der qualitative Nachweis von Halogen nach der Behandlung unzweifelhaft mit ausgezeichneter Schärfe. Da infolge des sorgfältigen Auswaschens eine Täuschung durch einfach gelöste Salzsäure oder Chlorkalk ausgeschlossen ist, so wird unsere oben ausgesprochene Anschauung, daß es sich bei unserem Reinigungsverfahren im wesentlichen um eine Umwandlung ungesättigter Verbindungen in gesättigte handelt, berechtigt sein. Es soll dabei nicht in Abrede gestellt werden, da $B$ durch unser Reinigungsverfahren noch ein Teil der ungesättigten Verbindungen durch Oxydation entfernt worden ist.

Die mit dem so gereinigten Ole angestellten Tierversuche zeigten, daß es auf diesem Wege gelungen war, die reizenden Substanzen in erheblichem Grade zu entfernen.

So wiesen die mit dem gereinigten Öle eingeriebenen Kaninchenohren den mit ungereinigtem ÖI bestrichenen gegenüber nur nach längerer Einreibung Rötungen auf. Exsudationen waren nicht zu bemerken. Allerdings verhielten sich die verschiedenen uns iibersandten Ölproben nicht ganz gleich.

Während es gelang, aus einigen verhältnismäßig leicht mit der Chlorbehandlung die reizenden Substanzen zu entfernen, war das bei später gelieferten Proben nicht in vollem Maße möglich. Immerhin war im Vergleich zu den Kontrollen, die ungereinigtes öl bekommen hatten, stets eị recht beträchtlicher Reinigungseffekt festzustellen.

Selbstverständlich kann die Haut unserer albinotischen Kaninchen nicht ohne weiteres mit menschlicher Haut verglichen werden, jedenfalls war sie viel empfindlicher als diese. So konnten in unseren Betrieben ekzematöse Prozesse, die auf eine.erheblichere Entzündung der tieferen Gewebspartien der mensehlichen Haut hätten schließen lassen, bei Ver- 
wendung der ungereinigten Öle nicht beobachtet werden. Bei den von uns beobachteten Arbeitern handelte es sich nur um Akneformen.

Um den Erfolg der Chlorkalkbehandlung in etwas gröBerem. Maßstabe prüfen zu können, wurden von einer neuen Ölsendung $11 / 2$ Liter in Arbeit genommen. Das öl hatte einen etwas weniger unangenehmen und weniger intensiven Geruch. Um die Säurezahl zu bestimmen; wurden $5 \mathrm{ccm}$ in einem Gemisch von $30 \mathrm{ccm}$ Alkohol in $30 \mathrm{ccm}$ Äther gelöst. Nach Zusatz von Phenolphthalein blieb diese bräunliche Lösung unverändert, ein bis zwei Tropfen Normalkalilauge riefen dann dagegen intensive Rotfärbung hervor. Das Öl war also gegen Phenolphthalein so gut wie neutral.

Das Rohöl wirkte auf Albinoohren sehr bald schädigend ein. Um das Verhältnis des Chlorkalkes zum Öl, das bei dem ersten Versuch ein gutes Resultat gegeben hatte, beizubehalten, wurden 1.5 Liter des Rohöls in einem großen hohen Glaszylinder mit $450 \mathrm{~g}$ Chlorkalkpulver versetzt. Der Chlorkalk wurde in kleinen Mengen eingetragen und jeweils kräftig durchgeschüttelt. Nachdem alles zugegeben war, ließen wir unter häufigem kräftigen Schütteln 2 .Tage lang bei Zimmertemperatur den Chlorkalk auf das ÖI einwirken, möglichst gut absitzen und filtrierten dann das öl durch ein Faltenfilter ab. Das Öl war klar, schied aber nach längerem Stehen einen fein verteilten, schwarzen, harzigen Niederschlag ab, von dem nochmals abfiltriert wurde. Von einem Waschen mit Wasser wurde abgesehen.

Das so behandelte Öl schädigte das Ohr der Albinos bedeutend weniger als das Rohöl. Leider war aber diese Chlorkalkbehandlung recht verlustreich; da der Chlorkalk sehr viel von dem dickflüssigen Öl festhielt, wurden nur zwei Drittel des angewendeten Öles wieder gewonnen. Dazu kommt noch, daB bei dem heutigen Mangel an Chlorkalk die Anwendung des Verfahrens im Großen in Frage gestellt wird.

Es wurde deshalb versucht, ob nicht wässerige Natriumhypochloridlösung, die in der Technik ja neuerdings viel Verwendung findet, für unsere Zwecke an Stelle des Chlorkalks treten könnte.

Wir stellten uns eine ziemlich konzentrierte Na-Hypochloridlösung durch Einleiten von Chlor in eine fünffach Normalnatronlauge, bis letztere fast gesättigt war, dar. Mit dieser Hypochloridlauge wurde das Rohöl gut durchgeschüttelt. Merkwürdigerweise verschwand der charakteristische Geruch des Rohöls nur äußerst langsam und blieb auch bestehen bei Verwendung eines großen Uberschusses von Hypochlorid.' Das Rohöl war zunächst mit kleineren Mengen Hypochloridlauge geschüttelt worden, schließlich war der Verbrauch an Lauge im Verhältnis zum Öl zwei zu eins.

Das Öl wurde dann im Scheidetrichter von der Lauge getrennt, mit Wasser gut ausgewaschen, mit Chlorkalzium getrocknet und filtriert. 
Die Prüfung fiel auffallenderweise weniger befriedigend aus, als nach der Chlorbehandlung, was sich auch nicht änderte, als die Behandlung desselben Öls mit noch mehr Hypochlorid wiederholt wurde.

Eine direkte Chlorbehandlung war bisher nicht unternommen worden, da ihre Anwendung für nicht gesehulte Arbeiter an den Verbrauchsstätten des Öls und ohne geeignete Hilfsmittel nicht zweckmäßig erschien. Ein Versuch im Kleinen führte jedoch zu so günstigem Erfolg, daß darüber berichtet werden soll.

In $400 \mathrm{ccm}$ Rohöl wurde einige": Minuten lang Chlorgas eingeleitet. Dabei erwärmte sich das Öl, die Wandung des Glaskolbens bedeckte sich mit einer mäßigen Harzschicht und Salzsäure entwich. Sobald diese Salzsäureentwicklung stärker wurde, wurde der Chlorstrom abgestellt, das öl vom Harz abfiltrirt, dann im Scheidetrichter wiederholt mit viel Wasser ausgewaschen und mit Chlorkalzium getrocknet.

Dies so behandelte Öl blieb auch nach mehrtägigem Stehen klar, war fast geruchlos. Der Gehalt an Chlorverbindungen war, nach der Flammenfärbung zu urteilen, nur gering. Die Prüfung war durchaus befriedigend.

Während bei den Kontrolltieren schon nach wenigen Einreibungen recht bemerkenswerte Entzündungen wahrzunehmen waren, zeigten sich an den Ohren der mit dem gereinigten Öle behandelten Tiere erst viel später entzündliche Frscheinungen in geringerem Grade.

Aus unseren Versuchen können allgemeingültige Vorschriften für die Reinigung derartiger Öle selbstverständlich nicht abgeleitet werden. Die Zusammensetzung der Öle ist eine zu wechselnde, so daß seitens der liefernden Firmen stets die jeweils passende Reinigungsmethode für eine größere Lieferung ausprobiert werden muß. Da bei dem jetzigen Ölmangel und wegen der Schwierigkeit der Beschaffung geeigneter Chomikalion in absehbarer Zeit öle mit reizenden Substanzen voraussichtlich in der Praxis vielfach in Kouf genommen werden müssen, haben wir nach anderer Richtung den Betrieben durch Bereitstellen geeigneter Schutzeinrichtungen zu nutzen gesucht.

Die von einem Betrieb vor unserem Eingreifen verwendeten Schutzärmel waren ganz unzweckmäßig, ja verschlimmerten das Ubel nur, da sie sich mit dem Öle vollsaugten, so daß die Berührung der Haut mit den reizenden Substanzen eine noch innigere wurde.

Wir bemühten uns deshalb, einen gegenuiber dem öl widerstandsfähigen, undurchlässigen Stoff ausfindig zu machen. Der nach dem Verfahren von Dr. Eichengrün mit Cellonlack imprägnierte Ballonstoff, wie er von der Firma Riedinger, Augshurg. benutzt wird, erwies sich 
in unseren Vorversuchen als geeignct. ${ }^{1}$ Die genannte Firma stellte uns verschiedene Proben zur Verfügung, deren Widerstandsfähigkeit gegenüber dem Öl wir durch längere Behandlung feststellten. Eine dieser Proben erwies sich als besonders widerstandsfähig. Aus dieser ließen wir zunächst Schutzärmel anfertigen, die beim Arbeiten an den betreffenden Maschinen verwendet wurden.

Allerdings haben Schutzärmel an sich Nachteile, auf die mehrfach in der Literatur hingewiesen worden ist. Sie passen meist schlecht und hindern deshalb bei den Hantierungen. Verwendet man undurchlässige Stoffe, so wird die Hautausdünstung gehemmt, was bei besonders hoher Aubentemperatur von vielen Personen sehr lästig empfunden wird. Größere Hautpartien mit undurchlässigen Stoffen zu bedecken, ist vollkommen unzweckmäßig. Dagegen ist es angängig, die am meisten der Schädlichkeit ausgesetzten Hautpartien, wie die Vorderarme, durch gutpassende Schutzärmel und die Vorderseite des Körpers durch eine Schürze aus geeeignetem Stoffe zu schützen.

Die aus dem beschriebenen Material hergestellten Schutzärmel zeigten beim Arbeiten art den betreffenden Maschinen genügende Dauerhaftigkeit. Allerdings müssen derartige Schutzärmel für den Einzelnen nach MaB angefertigt werden. Sind die Ärmel zu bauschig, so hindern sie bei der Arbeit, ebenso wenn sie zu eng sind. Ferner müssen die Ärmel an den Gelenken gut abschließen, weil sonst nach längerem Gebrauche das Öl auch in das Innere des Ärmels gelangt. Ein umgelegtes Gummiband kann den Abschluß nach unten vervollständigen. Am Oberarm befestigten wir den Ärmel durch eine Knöpfvorrichtung.

Während einige Arbeiter von den Ärmeln befriedigt waren und noch einen erweiterten Schutz aus dem gleichen Material in Form einer Schürze wiinschten, wurden sie von anderen, vor allem während der heißen Sommertage, lästig empfunden. Fine Auswahl des geeigneten Personals seitens der Fabrikleitung, verbunden mit möglichster Reinigung der am meisten betroffenen Hautstellen nach der Arbeit und in den Betriebspausen wird, wenn wirksame Reinigangsverfahren der Öle nicht angewendet werden können, wesentlich dazu beitragen, die beobachteten Belästigungen auf ein erträgliches Maß herabzumindern.

1 Auf diesen Stoff hat uns Herr Prof. Busch aufmerksam gemacht. Ihm und Herrn Dr. Bernkopf, der uns als Facharzt für Dermatologie unterstützte, verdanken wir wertvolle Ratschläge. 